\title{
CONCERNING COLLECTIONS OF CUTTINGS OF CONNECTED POINT SETS*
}

\author{
BY G. T. WHYBURN
}

Two point sets are mutually separated if they are mutually exclusive and neither of them contains a limit point of the other. A point set $M$ is said to be not connected or connected according as $M$ is or is not the sum of two non-vacuous mutually separated point sets. A subset $X$ of a connected point set $M$ is said to be a cutting of $M$, or is said to cut $M$, provided the set of points $M-X$ is not connected; $X$ is called an irreducible cutting of $M$ provided $X$ cuts $M$ but no proper subset of $X$ cuts $M$. If $A$ and $B$ are subsets of a connected point set $M$, the subset $X$ of $M$ is said to separate $A$ and $B$ in $M$, or to cut $M$ between $A$ and $B$, provided that $M-X$ is the sum of two mutually separated sets $M_{a}(X)$ and $M_{b}(X)$ containing $A$ and $B$ respectively.

In this paper the ordinary notation of point set theory will be employed, for example, $\bar{X}=X+X^{\prime}$, where $X^{\prime}$ is the set of all limit points of the set $X, K \subset H$ means that the set $K$ is a subset of the set $H, K \cdot H$ means the set of points common to $K$ and $H, F(R)$ denotes the boundary of the set $R$, etc. In addition, if $X$ is a cutting of a connected point set $M$ then, unless otherwise stated, the equation $M-X=M_{a}(X)$ $+M_{b}(X)$ is to be interpreted as meaning that $M-X$ is the sum of two mutually separated sets $M_{a}(X)$ and $M_{b}(X)$ which contain the sets $A$ and $B$ respectively.

1. Cuttings of Connected Sets in General. The point sets considered in this section are assumed to lie in a separable metric space which will be denoted by $S$. It is apparent from the proofs of some of the theorems, however, that they hold in even more general space.

* Presented to the Society, April 7 and June 2, 1928. 
THEOREM 1. Let G be any uncountable collection of mutually exclusive cultings of a connected and separable* point set $M$. Then there exist two points $A$ and $B$ of $M$ which are separated in $M$ by uncountably many elements of $G$.

Proof. Since $M$ is separable, it therefore contains a countable set of points $D$ such that $M$ is a subset of $\bar{D}$. As $G$ is uncountable and $D$ is countable, there exists an uncountable subcollection $G_{1}$ of $G$ such that no element of $G_{1}$ contains a point of $D$. Now if $X$ is any element of $G_{1}, M-X$ is the sum of two mutually separated sets $M_{1}(X)$ and $M_{2}(X)$. Let $P_{1}$ and $P_{2}$ be points of $M_{1}(X)$ and $M_{2}(X)$ respectively. Since $P_{1}$ is a limit point of $D$ and is not a limit point of $M_{2}(X)$, then $D$ is not a subset of $M_{2}(X)$. And since $D \cdot X=0, D$ is not a subset of $M_{2}(X)+X$. Therefore $M_{1}(X)$ contains at least one point $A(X)$ of $D$. Similarly, $M_{2}(X)$ contains at least one point $B(X)$ of $D$. Thus every element $X$ of $G_{1}$ separates some pair of points $A(X)$ and $B(X)$ of $D$ in $M$. And since the elements of $G_{1}$ are uncountable and the collection of all pairs of points of $D$ is countable, it follows that some two points $A$ and $B$ of $D$ are separated in $M$ by uncountably many elements of $G_{1}$.

THEOREM 2. If $G_{0}$ is any collection of mutually exclusive subsets of a connected and separable set $M$ each of which contains a cutting of $M$, then all save possibly a countable number of the elements of $G_{0}$ must be cuttings of $M$.

Proof. Since $M$ is separable, it contains a countable set of points $D$ such that $M \subset \bar{D}$. By hypothesis each element $g$ of $G_{0}$ contains a cutting $X_{0}$ of $M$. Now let $G$ be the collection of all those elements of $G_{0}$ which are not cuttings

* It follows by a theorem of W. Gross (See Zur Theorie der Mengen, in denen ein Distanzbegriff definiert ist, Wiener Sitzungsberichte, vol. 123 (1914), pp. 801-819) that every subset of a separable metric space is separable. Hence that $M$ is separable follows from the fact that it is imbedded in the space $S$. However, the condition that $M$ be separable is explicitly stated in this theorem and in some of the theorems that are to follow because the separability of $M$ is used in the proof and because as thus stated the theorem holds in a more general space than the space $S$. 
of $M$. For each element $g$ of $G, M-X_{0}$ is the sum of two mutually separated sets $M_{1}$ and $M_{2}$; and since $M-g$ is connected and a subset of $M-X_{g}$, it therefore is a subset either of $M_{1}$ or of $M_{2}$, say of $M_{1}$. Then obviously $M_{2}$ is a subset of $g$. Let $P$ be a point of $M_{2}$. Then since $P$ is a limit point of $D$ but not of $M_{1}, D$ is not a subset of $M_{1}$. Hence $M_{2}+X_{0}$ contains at least one point of $D$; and as $M_{2}+X_{0}$ is a subset of $g$, then $g$ contains at least one point of $D$. Thus every element of $G$ contains at least one point of $D$. And since the elements of $G$ are mutually exclusive and $D$ is countable, it follows that $G$ is countable.

Theorem 3. If $X$ is any cutting of a connected set $M$ between two points $A$ and $B$ of $M$, and $K$ is any subset of $M$ which contains neither $A$ nor $B$, then $X+K$ cuts $M$ between $A$ and $B$.

Proof. By hypothesis $M-X=M_{a}(X)+M_{b}(X)$. Then $M-(X+K)=\left[M_{a}(X)-M_{a}(X) \cdot K\right]+\left[M_{b}(X)-M_{b}(X) \cdot K\right]$, and clearly $\left[M_{a}(X)-M_{a}(X) \cdot K\right]$ and $\left[M_{b}(X)-M_{b}(X) \cdot K\right]$ are mutually separated and contain $A$ and $B$ respectively.

THEOREM 4. If $N$ is any connected subset of a connected and separable set $M$, then not more than a countable number of the components* of $M-N$ can contain cuttings of $M$.

Proof. By a theorem of Knaster and Kuratowski's, $\dagger$ no component of $M-N$ can cut $M$. Therefore by Theorem 2 , not more than a countable number of the components of $M-N$ can contain any subset which cuts $M$.

THEOREM 5. If $G_{0}$ is a collection of mutually exclusive connected subsets of a connected and separable set $M$ such that for each element $g$ of $G_{0}, M-g$ is neither connected nor the sum of two connected sets, then $G_{0}$ is countable. $\neq$

* A component of a point set $K$ is a connected subset of $K$ which is not a proper subset of any connected subset of $K$. See Hausdorff, Mengenlehre.

$\dagger$ Sur les ensembles connexes, Fundamenta Mathematicae, vol. 2 (1921), pp. 206-253, Theorem 10.

$\ddagger$ This theorem is a generalization of a theorem due to Kuratowski and Zarankiewicz (see $A$ theorem on connected point sets, this Bulletin, vol. 33 
Proof. Suppose, on the contrary, that $G_{0}$ is uncountable. Then by Theorem 1 , there exist two points $A$ and $B$ of $M$ and an uncountable subcollection $G$ of $G_{0}$ each element of which separates $A$ and $B$ in $M$. Since $M$ is separable, it contains a countable set of points $D$ such that $M \subset \bar{D}$. For each element $g$ of $G, M-g$ is the sum of three mutually separated sets $M_{a}, M_{b}$, and $M_{a}$, where $M_{a}$ and $M_{b}$ contain $A$ and $B$ respectively. For each element $g$ of $G$, it follows as in the preceding proofs that $M_{0}+g$ contains at least one point of $D$. And since, ${ }^{*}$ for each $g, M_{a}+M_{b}+g$ is connected, and contains both $A$ and $B$, it therefore contains every element of $G$. Hence for every two distinct elements $X$ and $Y$ of $G, M_{x}+X$ and $M_{y}+Y$ are mutually exclusive sets; and as each such set contains a point of $D$, and $D$ is countable, it follows that $G$ is countable, contrary to supposition. Thus the supposition that Theorem 5 is false leads to a contradiction.

Let $A$ and $B$ be two connected subsets (or points) of a connected point set $M$, and let $G$ be any collection of mutually exclusive connected subsets of $M$ each of which separates $A$ and $B$ in $M$. Then the elements of $G$ can be linearly ordered in $M$ from $A$ to $B$ as follows. The element $X$ of $G$ will be said to precede or to follow the element $Y$ of $G$ in $M$ in the order from $A$ to $B$ according as $X$ belongs to $M_{a}(Y)$ or to $M_{b}(Y)$, where $M_{a}(Y)+M_{b}(Y)$ is one method of expressing $M-Y$ as the sum of two mutually separated sets containing $A$ and $B$ respectively. It is readily deduced from this definition that (a) of two elements $X$ and $Y$ of $G$, one must always precede the other, (b) $X$ cannot both precede and follow $Y$, and (c) if $X$ precedes $Y$, then $Y$ follows $X$.

(1927), p. 574), who assume the unnecessary condition that the elements of $G_{0}$ are closed relative to $M$. Although the proof here given for Theorem 5 differs markedly from that given by Kuratowski and Zarankiewicz to prove a special case of their theorem, I have found that a proof can be constructed for Theorem 5 based on their methods which is somewhat shorter than the proof they gave in the paper just mentioned and which makes no use of the fact that the set $M$ itself is separable.

* See Knaster and Kuratowski, loc. cit., Theorem 6. 
If $X$ and $Y$ are distinct elements of $G$ and $X$ precedes $Y$ in $M$ in the order from $A$ to $B$, then by the segment $S(X, Y)$ of $M$ from $X$ to $Y$ is meant the set of points $M_{b}(X) \cdot M_{b}(Y)$, where $M_{a}(X)+M_{b}(X)$ is one way of expressing $M-X$ as the sum of two mutually separated sets containing $A$ and $B$ respectively and similarly for $M_{a}(Y)$ and $M_{b}(Y)$; by the interval $I(X, Y)$ of $M$ from $X$ to $Y$ is meant the set of points $\left[M_{b}(X)+X\right] \cdot\left[M_{a}(Y)+Y\right]$. Clearly $I(X, Y)=S(X, Y)+$ $X+Y$. In case all of the sets $M_{a}(X), M_{b}(X), M_{a}(Y)$, and $M_{b}(Y)$ are connected, as is necessarily the case for all save possibly a countable number of elements of $G$ (see Theorem 5), then $S(X, Y)$ and $I(X, Y)$ are uniquely determined. The following facts are readily proved.

(i). For each pair of elements $X$ and $Y$ of $G$, where $X$ precedes $Y, M$ is the sum of three connected point sets $M_{a}(X)+X, I(X, Y)$, and $M_{b}(Y)+Y$, where we have $\left[M_{a}(X)+X\right] \cdot I(X, Y)=X,\left[M_{b}(Y)+Y\right] \cdot I(X, Y)=Y$, and $\left[M_{a}(X)+X\right] \cdot\left[M_{b}(Y)+Y\right]=0$.

(ii). No point of $S(X, Y)$ is a limit point of $M-I(X, Y)$.

(iii). If $M_{a}(X), M_{b}(X), M_{a}(Y)$, and $M_{b}(Y)$ are connected, and $S(X, Y)$ contains an element of $G$, then $S(X, Y)$ is connected. It may happen that for some element $X$ of $G$, an element $Y$ of $G$ exists such that $S(X, Y)$ is vacuous. However, Theorem 6 below shows that this can be the case for at most a countable number of elements $X$ of $G$.

THEOREM 6. Let $G$ be any uncountable collection of mutually exclusive connected cuttings of a connected set $M$. Then there exists a subcollection of $G^{*}$ of $G$, containing all but a countable number of the elements of $G$, and such that every two elements of $G^{*}$ are separated in $M$ by uncountably many elements of $G .{ }^{*}$

Proof. Since $M$ is separable, it contains a countable subset $D$ such that $M \subset \bar{D}$. Let $G_{1}$ denote the collection obtained by omitting from the collection $G$ (1) all elements $X$ of $G$ such that $M-X$ is not the sum of two connected point sets, (2) all elements of $G$ which contain at least one

† See Gross, loc. cit. 
point of $D$, and (3) all elements $X$ of $G$ which separate in $M$ some two points of $D$ which are separated in $M$ by only a countable number of elements of $G$. Then $G_{1}$ contains all but a countable number of the elements of $G$. Let $H$ denote the collection of all possible pairs of points of the set $D$ having the property that each pair $A, B$ in $H$ is separated in $M$ by each element of an uncountable subcollection $G_{a b}$ of the collection $G_{1}$. For each pair $A, B$ in $H$, let $E_{a b}$ denote the collection of all those elements $e$ of $G_{a b}$ such that every neighborhood of $e$ contains points of uncountably many elements of $G_{a b}$. By a theorem due to Gross (loc. cit.), $E_{a b}$ contains all but a countable number of the elements of $G_{a b}$. Hence every neighborhood of each element $e$ of $E_{a b}$ contains points of uncountably many elements of $E_{a b}$.

Now for each pair of elements $X$ and $Y$ of $E_{a b}, S(X, Y)$ is non-vacuous. For suppose $S(X, Y)$ is vacuous, and suppose $X$ precedes $Y$ in $M$ in the order from $A$ to $B$. Now $X$ contains a point $P$ which is a limit point of $M_{b}(X)$. And since $P$ is not a limit point of $M_{b}(Y)$ [for $X$ belongs to $M_{a}(Y)$ ], there exists a neighborhood $V$ of $P$ which contains no point of $M_{b}(Y)$. Now $V$ contains at least one point $Q$ of $M_{b}(X)$; and since $M_{b}(X)=M_{b}(Y)+Y+S(X, Y)$, and $S(X, Y)$ is vacuous, then $Q$ must belong to $Y$. Now $Q$ is not a limit point of $M_{a}(X)$, for $Q$ belongs to $Y$ and $Y$ belongs to $M_{b}(X)$. Hence there exists a neighborhood $U$ of $Q$ which contains no point of $M_{a}(X)$ and which is a subset of $V$. Then $U \cdot M$ is a subset of $X+Y$. But $U \cdot M$ must contain at least one point of $D$; and since $X$ and $Y$ belong to $G_{1}$, neither of them can contain a point of $D$. Thus the supposition that $S(X, Y)$ is vacuous leads to a contradiction.

Now the number of elements $X$ of $E_{a b}$ such that an element $Y$ of $E_{a b}$ exists such that $S(X, Y)$ contains no element of $E_{a b}$ must be countable. For each such segment $S(X, Y)$ must contain at least one point $P$; and since $P$ is a limit point of $D$ but not of $M-I(X, Y)$, and neither $X$ or $Y$ contains a point of $D$, then $S(X, Y)$ must contain at least one point of $D$. And clearly if $S\left(X_{1}, Y_{1}\right)$ and $S\left(X_{2}, Y_{2}\right)$ are two 
such segments which are different, they must be mutually exclusive. Therefore the number of such segments must be countable, and hence all save a countable number of elements $X$ of $E_{a b}$ must have the property that if $Y$ is any other element whatever of $E_{a b}$, then $S(X, Y)$ contains at least one element $Z$ of $E_{a b}$. Let $G_{a b}^{*}$ denote the collection of all those elements of $E_{a b}$ which have this property. Then if $X$ and $Y$ are any two elements of $G_{a b}^{*}, S(X, Y)$ contains at least one element $Z$ of $E$; and since every neighborhood of $Z$ contains points of uncountably many elements of $E_{a b}$, since no point of $Z$ is a limit point of $M-I(X, Y)$, and since $G_{a b}^{*}$ contains all but a countable number of the elements of $E_{a b}$, it follows that $S(X, Y)$ contains uncountably many elements of $G_{a b}^{*}$.

Let $G^{*}$ denote the collection of all those elements $g$ of $G$ such that $g$ belongs to some collection $G_{a b}^{*}$. Since $H$ is countable, it follows that $G^{*}$ contains all save a countable number of the elements of $G$. Now let $X$ and $Y$ be any two distinct elements of $G^{*}$. Then $M-X=M_{y}+M_{1}$, where $M_{y}$ and $M_{1}$ are mutually separated and $M_{y}$ contains $Y$. It is readily seen that $M_{1}$ contains at least one point $A$ of $D$. Likewise $M-Y=M_{x}+M_{2}$, and $M_{2}$ contains a point $B$ of $D$. It is readily seen that both $X$ and $Y$ separate $A$ and $B$ in $M$. Since $X$ and $Y$ belong to $G_{1}$, then $A$ and $B$ are separated in $M$ by uncountably many elements of $G$. Hence $A, B$ is a pair in $H$, and $X$ and $Y$ belong to $G_{a b}^{*}$. Therefore $S(X, Y)$ contains uncountably many elements $[Z]$ of $G_{a b}^{*}$. Since $M_{a}(X)+X$ and $M_{b}(Y)+Y$ are connected and contain $A$ and $B$ respectively but contain no point of $Z$, for each $Z$ in $[Z]$, then $X$ belongs to $M_{a}(Z)$ and $Y$ to $M_{b}(Z)$. Hence $Z$ separates $X$ and $Y$ in $M$. Thus every two elements $X$ and $Y$ of $G^{*}$ are separated in $M$ by uncountably many elements of $G,^{*}$ and our theorem is proved.

THEOREM 7. If $G^{*}$ is the collection obtained in Theorem 6 and $E$ is the collection of elements $e$ such that $e=\bar{g} \cdot M$, for

\footnotetext{
† See Knaster and Kuratowski, loc. cit., Theorem 6.
} 
each element $g$ of $G,{ }^{*}$ then the elements of $E$ are mutually exclusive, all save a countable number are cuttings of $M$ and every two of them are separated in $M$ by some third element of $E$ (indeed, by uncountably many elements of $E$ ).

Proof. Let $d$ and $e$ be two distinct elements of $E$, where $d=\bar{g} \cdot M$ and $e=\bar{h} \cdot M$, where $g$ and $h$ are elements of $G^{*}$. There exists an element $i$ of $G^{*}$ such that $M-i=M_{\sigma}+M_{h}$. Hence $h$ contains no limit point of $g$; similarly it is shown that $i$ contains no limit point of either $g$ or $h$. Then since $d$ and $e$ are subsets of $M_{g}+i$ and $M_{h}+i$ respectively, and $d \cdot i=e \cdot i=0$, it follows that $d$ and $e$ are subsets of $M_{\vartheta}$ and $M_{h}$ respectively. Hence $d$ and $e$ are mutually exclusive. A similar argument shows that if $f$ is the element of $E$ such that $f=i \cdot M$, then $f$ separates $d$ and $e$ in $M$. That all save possibly a countable number of the elements of $E$ are cuttings of $M$ follows now from Theorem 2. The truth of Theorem 7 is therefore established.

2. Cuttings of Continua. In this section the point sets considered are assumed to lie in a locally compact, metric, and separable space, that is, the same space considered in $\$ 1$ with the additional restriction that it be locally compact.

THEOREM 8. No continuum $M$ contains an uncountable collection $G$ of mutually exclusive connected subsets each of which contains a compact proper subset which cuts $M . \dagger$

Proof. Suppose, on the contrary, that some continuum $M$ contains such a collection $G$. Each element $g$ of $G$ contains a closed $\ddagger$ and compact set $f$ which cuts $M$ and a point

$\dagger$ For the special case where the continuum $M$ is identical with a whole euclidean space of $n$ dimensions, this theorem is readily deduced from a theorem of Kuratowski's; see C. Kuratowski, Sur les coupures irréductibles du plan, Fundamenta Mathematicae, vol. 6 (1924), pp. 130-146, Theorem 8.

$\ddagger$ For justification of the word "closed" here see R. L. Moore (Proceedings of the National Academy of Sciences, vol. 10 (1924), pp. 356-360), who proves, with the aid of a theorem of Knaster and Kuratowski (Sur les ensembles connexes, loc. cit., Theorem 37), that every cutting of a bounded plane continuum $M$ between two points $A$ and $B$ of $M$ contains a closed cutting of $M$ between $A$ and $B$. Although the theorem of Knaster 
$p$ not belonging to $f$. For each element $g$ of $G$ select two such sets $f$ and $p$, let $F$ denote the collection of sets $[f]$, and let $P$ denote the set of points $[p]$. For each element $g$ of $G$, let $\delta(f, p)$ be the minimum distance between the sets $f$ and $p$. There exists a number $\epsilon>0$ and an uncountable subcollection $G_{1}$ of $G$ such that for each element $g_{1}$ of $G_{1}, \delta\left(f_{1}, p_{1}\right)$ $>4 \epsilon$. Let $F_{1}$ and $P_{1}$ denote the collections $\left[f_{1}\right]$ and $\left[p_{1}\right]$, for all elements $g_{1}$ of $G_{1}$. There exists a point $p$ which is a point of condensation of $P_{1}$. Let $R$ be the set of all points whose distance from $p$ is less than $\epsilon$. Then no element of $F_{1}$ contains a point of $\bar{R}$. Let $P_{2}$ be the set of points $R \cdot P_{1}$, and let $F_{2}$ and $G_{2}$ be the corresponding subcollections of $F_{1}$ and $G_{1}$ respectively, that is, each point $p_{2}$ of $P_{2}$ corresponds to an element $f_{2}$ in $F_{2}$ and an element $g_{2}$ in $G_{2}$. By a theorem* established in its most general form by R. L. Moore, there exists an element $f$ of $F_{2}$ such that every neighborhood of $f$ contains uncountably many elements of $F_{2}$. Since $f$ is compact, it follows with the aid of the Borel theorem that there exists a compact open set $D$ which contains $f$ but contains no point whatever of $R$. Let $F_{3}$ be the collection of all those elements of $F_{2}$ which lie wholly in $D$, and let $G_{3}$ be the corresponding collection of elements of $G_{2}$.

By Theorems 1, 3, and 6 it follows that there exist two points $A$ and $B$ of $M$ and an uncountable subcollection $G_{4}$ of $G_{3}$ such that if $F_{4}$ is the corresponding collection of elements of $F$, then (1) each element of $F_{4}$ and also each element of $G_{4}$ separates $A$ and $B$ in $M$, and (2) every two elements $X$ and $Y$ of $G_{4}$ are separated in $M$ by uncountably

and Kuratowski used by Moore does not necessarily remain true in the space considered in this paper, nevertheless the methods of proof used by Knaster and Kuratowski in proving their theorem suffice to prove Moore's theorem in such a space, and indeed to prove the following more general theorem: If $K$ is a cutting of a continuum $M$ (bounded or not) between any two subsets $A$ and $B$ of $M$, then $K$ contains a closed cutting of $M$ between $A$ and $B$.

* See R. L. Moore, Concerning triodic continua in the plane, to appear in Fundamenta Mathematicae; for the case of a euclidean space see a theorem of C. Zarankiewicz in his paper Sur les points de division dans les ensembles connexes, Fundamenta Mathematicae, vol. 9 (1927), Theorem 2. 
many elements of $G^{4}$. Let $T$ denote the point set consisting of the sum of all the point sets of the collection $G_{4}$, and for each element $g$ of $G_{4}$, let $e$ denote the component of $M-$ $(T-g)$ which contains $g$, and let $E$ denote the collection of sets $[e]$ thus obtained. Then each element $e$ of $E$ must be closed; for if some element $h$ of $G_{4}$, different from the element of $G_{4}$ which belongs to $e$, contained a limit point of $g$, then $e+h$ would be connected and would contain both $e$ and $h$, contrary to the fact that an element $j$ of $G_{4}$ exists which contains no point of $e+h$ but which separates $g$ and $h$ in $M$. Hence each element of $E$ is a continuum, and a similar argument shows that the elements of $E$ are mutually exclusive and that every two elements of $E$ are separated in $M$ by some third element of $E$. Since $E$ is uncountable, it follows readily from Theorems 3 and 5 together with the theorem of Moore-Zarankiewicz quoted above that there exists an element $e$ of $E$ such that (1) $e$ separates $A$ and $B$ in $M$ and both $M_{a}(e)$ and $M_{b}(e)$ are connected, (2) either every point of $e$ is a limit point of $M_{a}(e)$ or every point of $e$ is a limit point of $M_{b}(e)$, suppose the former, and (3) there exists a point $p$ of $e$ and a sequence of points $p_{1}, p_{2}, p_{0}, \cdots$, such that for each $i, p_{i}$ belongs to an element $e_{i}$ of $E$ which belongs to $M_{b}(e)$, and such that $p$ is the sequential limit point of the sequence of points $p_{1}, p_{2}, p_{3}, \cdots$. For each $i$, let $q_{i}$ denote the component of $e_{i} \cdot \bar{D}$ which contains $p_{i}$, and let $K$ denote the sequential limiting set of the sequence $q_{1}, q_{2}, q_{3}, \cdots$. By a theorem due to Janiszewski, ${ }^{*}$ for each $i$, $q_{i}$ is a compact continuum containing at least one point of $F(D)$. Hence by a theorem proved in its most general form by R. G. Lubben, $\uparrow$ it follows that $K$ is a continuum. Clearly $K$ contains at least one point $X$ of $F(D)$. Now $K$ contains no point of $T-e$. For suppose $K$ does contain a point $Y$ of $T-e$; then $Y$ belongs to some element $j$ of $E$; and

* Sur les continus irreductibles entre deux points, Journal de l'École Polytechnique, (2), vol. 16 (1912).

† See R. G. Lubben, Concerning limiting sets in abstract spaces, Transactions of this Society, vol. 30 (1928), pp. 668-685. 
there exists an element $d$ of $E$ such that $M-d=M_{e}(d)+$ $M_{j}(e)$. Either $M_{e}(d)$ or $M_{j}(e)$ must contain infinitely many elements of the sequence $q_{1}, q_{2}, q_{3}, \ldots$. But $\mathrm{M}_{e}(d)$ cannot contain infinitely many elements of this sequence; for if it did, then $Y$, a point of $M_{j}(d)$, would be a limit point of $M_{e}(d)$. Likewise $M_{j}(d)$ cannot contain infinitely many elements of this sequence; for if so, then $p$, a point of $M_{e}(d)$, would be a limit point of $M_{j}(d)$. Thus the supposition that $K$ contains a point of $T-e$ leads to a contradiction. Then since $K$ is a connected subset of $M$ which contains $p$ but contains no point of $T-e$, and $e$ is the component of $M-$ $(T-e)$ which contains $p, K$ must be a subset of $e$. Hence $e$ contains the point $X$. Then $X$ is a limit point of $M_{a}(e)$, for every point of $e$ is a limit point of $M_{a}(e)$; and $X$ is also a limit point of $M_{b}(e)$. Hence $M_{a}(e)+X+M_{b}(e)$ is connected and contains both $A$ and $B$. But this is contrary to the fact that $e$ contains an element $f$ of $F$ which lies wholly in $D$ (and hence does not contain $X$ ) and which separates $A$ and $B$ in $M$. Thus the supposition that Theorem 8 is false leads to a contradiction.

Corollary. If $G$ is any collection of mutually exclusive connected subsets of a continuum $M$ each of which contains a compact cutting of $M$, then all save possibly a countable number of the elements of $G$ are themselves compact continua which are irreducible cuttings of $M$.

THEOREM 9. If $G$ is any collection of mutually exclusive connected subsets of a continuum $M$ each of which contains $a$ compact cutting of $M$, and $T$ denotes the point set obtained by adding together all the point sets of the collection $G$, then all save possibly a countable number of the elements of $G$ are components of $M-(T-g)$; indeed, all save a countable number of the elements $g$ of $G$ have the property that every connected subset of $M$ which contains $g$ but is not identical with $g$ must contain points of uncountably many elements of $G$.

Proof. By Theorems 6 and 8, $G$ contains a subcollection $G_{1}$ which contains all but a countable number of the elements 
of $G$ and such that the elements of $G_{1}$ are compact continua which are irreducible cuttings of $M$ and have the property that every two of them are separated in $M$ by uncountably many elements of $G_{1}$. Now let $E$ denote the collection of all those elements $g$ of $G_{1}$ such that $g$ is a proper subset of some connected subset $X$ of $M$ which has points in common with at most a countable number of elements of $G$. Let $[X]$ denote the collection of sets $X$. Then since every two elements of $G_{1}$ are separated in $M$ by uncountably many elements of $G_{1}$, it follows that no element of $[X]$ contains points of more than one element of $G_{1}$ and that no two elements of $[X]$ have a point in common. And since each element of $[X]$ contains a compact proper subset which cuts $M$, it follows by Theorem 8 that $[X]$ must be countable. Therefore $E$ is countable.

THEOREM 10. If $G_{0}$ is any uncountable collection of mutually exclusive connected subsets of a continuum $M$ each of which contains a compact cutting of $M$, then $G_{0}$ contains a subcollection $G$ which contains all save possibly a countable number of the elements of $G_{0}$ and has the following properties: (1) every element $g$ of $G$ is a compact continuum which is an irreducible cutting of $M$ and is a component of the set of points $M-(T-g)$, where $T$ denotes the point set obtained by adding together all the point sets of the collection $G,(2)$ every two elements of $G$ are separated in $M$ by uncountably many elements of $G$, and (3) $G$ is upper semi-continuous.*

Proof. That $G_{0}$ contains a collection $G$ having properties (1) and (2) follows immediately from Theorems 6, 8, and 9. I shall now show that any collection $G$ of mutually exclusive

* A collection $G$ of point sets is said to be upper semi-continuous provided that if $g$ is any element of $G$ and $g_{1}, g_{2}, g_{3}, \ldots$ is any infinite sequence of elements of $G$ containing points $P_{1}, P_{2}, \cdots$, respectively, such that the sequence of points $P_{1}, P_{2}, \cdots$ has a sequential limit point which belongs to $g$, then $g$ contains the entire sequential limiting set of the sequence of sets $g_{1}, g_{2}, g_{3}, \cdots$. See R. L. Moore, Concerning upper semi-continuous collections of continua, Transactions of this Society, vol. 27 (1925), pp. 416428. 
cuttings of $M$ having properties (1) and (2) must be upper semi-continuous. Let $g_{1}, g_{2}, g_{3}, \cdots$ be any sequence of elements of $G$ containing points $P_{1}, P_{2}, P_{3}, \cdots$, respectively, such that the sequence $P_{1}, P_{2}, P_{3}, \cdots$ has a sequential limit point $P$ belonging to some element $g$ of $G$. Let $L$ denote the sequential limiting set of the sequence $g_{1}, g_{2}, \ldots$. Then $g$ must contain $L$. For if not, then since $g$ is compact, it follows readily that $g$ is a proper subset of the component $K$ of $g+L$ which contains $g$. And, since $g$ is a component of $M-(T-g)$, $K$ must contain a point $Q$ of some other element $h$ of $G$ different from $g$. And by (2) there exists an element $f$ of $G$ which separates $g$ and $h$ in $M$, that is, $M-f=M_{g}(f)+M_{h}(f)$. Now either $M_{g}(f)$ or $M_{h}(f)$ must contain infinitely many sets of the sequence $g_{1}, g_{2}, \ldots$. But it is easy to see that neither of these sets can contain infinitely many sets of this sequence, because each of these sets contains a point of $L$. Hence $g$ contains $L$, and therefore $G$ is an upper semi-continuous collection.

Corollary. If $G$ is any collection of mutually exclusive compact subcontinua of a continuum $M$ such that (1) each element $g$ of $G$ is saturated with respect to the property of being a subcontinuum of $M-(T-g)$, where $T$ is the point set obtained by adding together all the point sets of the collection $G$, and (2) every two elements of $G$ are separated in $M$ by some third element of $G$, then $G$ is upper semi-continuous.

Suppose $M$ is a compact continuum and $G$ is a collection of mutually exclusive sets having properties (1) and (2) in the statement of Theorem 10. Then by property (1) it follows that every component of $M-T$ is closed and hence is a compact continuum. And if $G_{0}$ denotes the collection whose elements are the elements of $G$ together with all the components of $M-T$, then the sum of all the elements of $G_{0}$ is identical with $M$; and using property (2) of $G$ it is readily shown that $M$ is an acyclic continuous curve (that is, a connected im kleinen continuum which contains no simple closed curve) with respect to the elements of $G_{0}$, and thus $G_{0}$ is upper semi-continuaus. The same is true if instead of as- 
suming $M$ compact we assume merely that each component of $M-T$ is compact. Thus we have the following theorem.

THEOREM 11. If $M$ is any compact continuum and $G$ is any uncountable collection of mutually exclusive connected subsets of $M$ each of which contains a cutting of $M$, then there exists an upper semi-continuous collection $G_{0}$ of mutually exclusive compact subcontinua of $M$ such that (1) all save possibly a countable number of elements of $G$ are elements also of $G_{0}$, (2) the sum of all the elements of $G_{0}$ is identical with $M$, and (3) $M$ is an acyclic continuous curve with respect to the elements of $G_{0}$.

3. The Order of Various Cuttings and im Kleinen Cuttings of Continua.* With the aid of the results established above it follows that all of the theorems, except Theorem 10, in the author's paper Concerning the cut points of continua $\dagger$ hold true in any separable metric space which is locally compact. In particular, the theorem (loc. cit., Theorem 7) that all save possibly a countable number of the cut points of any continuum $M$ are points of Menger order two of $M$ holds true in such a space. Using the results proved above and a method essentially the same as that used to prove Theorem 7 in the paper of the author's just mentioned, it is not difficult to prove the following more general theorem.

THEOREM 12. If $M$ is any continuum and $G$ is any collection of mutually exclusive compact subcontinua of $M$ each of which cuts $M$, then all, save possibly a countable number, elements $X$ of $G$ have the property that for each $\epsilon>0$, an open set $R$ exists containing $X$ and every point of which is at a distance $<\epsilon$ from some point of $X$ and such that $F(R) \cdot M=Y+Z$, where $Y$ and $Z$ are continua of the collection $G$.

Thus all save possibly a countable number of elements of $G$ are continua of Menger order (or Urysohn index) two of

* The space considered in this section is the same as the one considered in $\$ 2$.

† Transactions of this Society, vol. 30 (1928), No. 3, pp. 597-609. 
$M$ in a certain generalized sense. Menger* and Urysohn* call a point $P$ of a continuum $M$ a point of "Verzweigungs" order (or index) $n$ of $M$ provided that for each $\epsilon>0, P$ can be $\epsilon$-separated $\dagger$ by $n$ subcontinua of $M$ but not by $n-1$ such continua, that is, for each $\epsilon>0$ an open set $R$ exists which contains $P$ and is of diameter $<\epsilon$ and such that $F(R) \cdot M$ contains at most $n$ components and such that $n$ is the least integer such that this property is preserved. If we extend this notion to include "continua $P$ " as well as "points $P$," and say that a subcontinuum $P$ of a continuum $M$ is $\epsilon-$ separated by $n$ subcontinua of $M$ provided that for each $\epsilon>0$ an open set $R$ exists which contains $P$ and such that (1) every point of $R$ is at a distance $<\epsilon$ from some point of $P$ and (2) $F(R) \cdot M$ has at most $n$ components, then Theorem 12 states that all save possibly a countable number of the elements of $G$ are continua of "Verzweigungs" order two of $M$, or indeed, that all save possibly a countable number of the elements of $G$ can, for each $\epsilon>0$, be $\epsilon$-separated by two continua which are elements of $G$.

If $C$ is a subcontinuum of a continuum $M$ such that for each $\epsilon>0$, an open set $R$ exists which contains $C$ and such that (1) every point of $R$ is at a distance $<\epsilon$ from some point of $C$ and (2) $C$ is a cutting of the component of $M \cdot \bar{R}$ which contains $C$, then $C$ will be called an im kleinen cut continuum of $M$. For the special case where $C$ is a single point, $C$ will be called an im kleinen cut point, or a cut point im kleinen, of $M$. The notion of a cut point im kleinen of a continuum is embodied in Urysohn's notion of an "unvermeidbar" (unavoidable) point of a continuum $\ddagger$ and in R. L. Moore's

* See K. Menger, Grundz üge einer Theorie der Kurven, Mathematische Annalen, vol. 95 (1925), pp. 276-306, and P. Urysohn, Über im kleinen zusammenhangende Kontinua, Mathematische Annalen, vol. 98 (1927), pp. 296-308, and earlier papers by the same authors referred to therein.

† See P. Urysohn, Sur la ramification des lignes Cantoriennes, Comptes Rendus, vol. 175 (1922), p. 481.

¥ See P. Urysohn, Über im kleinen zusammenhangende Kontinua, loc. cit.; and R. L. Moore, Concerning triods in the plane and the junction points 
notion of a "junction" point of a continuum. For the case of a continuous curve (that is, a connected im kleinen continuum) it has been observed by W. L. Ayres and the author that the notions of an unavoidable point in the sense of Urysohn and of an im kleinen cut point as above defined are equivalent.

THEOREM 13. If $H$ denotes the set of all the points of a continuum $M$ at which $M$ is connected im kleinen and $K$ denotes the set of all the im kleinen cut points of $M$, then all save possibly a countable number of the points of $K \cdot H$ are points of Menger order two of $M$.

Proof. Suppose, on the contrary, that $H \cdot K$ contains an uncountable subset $N$ no point of which is a point of order two of $M$. For each point $P$ of $N$ there exists a number $E_{p}>0$ such that if $R_{p}$ denotes the component of $M \cdot S\left(P, E_{p}\right)$ containing $P$, where $S\left(P, E_{p}\right)$ denotes the set of all points whose distance from $P$ is $\leqq E_{p}$, then $P$ is a cut point of $R_{p}$. There exists a number $e>0$ and an uncountable subset $N_{1}$ of $N$ such that for each point $P_{1}$ of $N_{1}, E_{p_{1}}>4 e$. There exists a point $Q$ of $N_{1}$ which is a point of condensation of $N_{1}$. Let $W$ denote the component of $M \cdot S(Q, e)$ which contains $Q$. Since $M$ is connected im kleinen at $Q$ and $Q$ is a point of condensation of $N_{1}$, there exists an uncountable subset $N_{2}$ of $N_{1}$ every point of which belongs to $W$ and is at a distance $<e / 2$ from $Q$. Then for each point $X$ of $N_{2}, R_{x}$ must contain $W$, because $E_{x}>4 e$. Hence, by a lemma of R. L. Moore's, ${ }^{*}$ each point of $N_{2}$ is a cut point of $W$. Then by the theorem of the author's stated in the first paragraph of this section it follows that there exists at least one point $Y$ of $N_{2}$ which is a point of Menger order two of $W$. And since $Y$ is not a limit point of $M-W$ (for $M$ is connected im kleinen at $Y$ ), then $Y$ is a point of order two of $M$, contrary to hypothesis. Thus the supposition that Theorem 13 is false leads to a contradiction.

of plane continua, Proceedings of the National Academy of Sciences, vol. 14 (1928), pp. 85-88.

* Concerning triods ..., loc. cit., Lemma 2. 
Corollary 1. All save possibly a countable number of the cut points im kleinen of any continuous curve $M$ are points of Menger order two of $M$.

COROLlary 2. The junction points* of any continuous curve are countable.

THEOREM 14. If every subcontinuum of a continuous curve $M$ contains uncountably many im kleinen cut points of $M$, then $M$ is a Menger regular curve. $\dagger$

Theorem 14 follows at once from Theorem 13 and Menger's theorem (loc. cit., Theorem 8) that the set of all non-regular points of any continuum either is vacuous or else contains a continuum. For the case where $M$ lies in the plane, Theorem 13 is related to Theorem 11 in the author's paper Concerning certain accessible points of plane continua $t$

* A junction point of a continuum $M$ is a cut point im kleinen of $M$ which is the emanation point of some triod which lies in $M$, where by a triod is meant a continuum which is the sum of three continua $A O, B O$ and $C O$, irreducible between the points $A$ and $O, B$ and $O$, and $C$ and $O$ respectively, and such that $O$ is the only point common to any two of them, and by the emanation point of a triod is meant the point corresponding to the point $O$ in the definition just given. (See R. L. Moore, Concerning triods..., loc. cit.) Moore shows that the junction points of any plane continuum are countable, whether $M$ is a continuous curve or not. That Moore's theorem is not true in 3-space is shown by the following example. Let $K$ be a non-dense perfect set on an interval $I$ of the $X$-axis. For each point $X$ of $K$, with $X$-coordinate $x$, let $T_{x}$ denote the triod which is the sum of the straight line interval joining the points $(x, 0,0)$ and $(x, 2,0)$ and the one joining $(x, 1,0)$ and $(x, 1,1)$. Let $M=I+\sum_{x \in h} T_{x}$. Then for each point $X$ of $K$, the point $(x, 1,0)$ is a junction point of the continuum $M$. Corollary 2 shows that the condition in Moore's theorem that the continuum $M$ lie in the plane may be replaced by the condition that $M$ be a continuous curve. Incidentally, Corollary 2 gives a more general result than the results of Wazewski-Menger and Alexandroff referred to in the introduction of the above mentioned paper of Moore's, even in $n$ dimensions or in any locally compact, metric, and separable space.

$\dagger$ A Menger regular curve is a continuum $M$ all of whose points are regular in the sense of Menger, that is, each point of $M$ can, for each $\epsilon>0$, be $\epsilon$-separated by a finite number of points of $M$; see K. Menger, Grundzuge einer Theorie der Kurven, loc. cit.

$\ddagger$ Monatshefte für Mathematik und Physik, vol.35 (1928), pp. 289-304. 
CoRollary. If every point of a continuous curve $M$ is a cut point im kleinen of $M$, (or is an unavoidable point of $M$ in the sense of Urysohn), then $M$ is a Menger regular curve.*

A continuous curve having the property stated in this corollary, that is, every one of its points is an im kleinen cut point, has a number of interesting simple properties, such as, for example, every subcontinuum of any one of its maximal cyclic curves $C$ contains an arc segment which is an open subset of $C$.

Using the theorems in the preceding sections together with methods similar to those used in the proof of Theorem 13 , it follows that if $G$ is any collection of mutually exclusive compact im kleinen cut continua of a continuous curve $M$, then all save possibly a countable number of the continua of $G$ are continua of "Verzweigungs" order two of $M$. Likewise if we follow R. L. Moore (loc. cit.) and call a subcontinuum $N$ of a continuum $M$ a junction continuum of $M$ provided $N$ is an im kleinen cut continuum of $M$ and is the emanation continuum of some analog of a triod $\dagger$ belonging to $M$, then no continuous curve contains an uncountable collection of mutually exclusive compact junction continua of itself.

For the case of the plane, it follows from the results of this paper by methods of proof essentially the same as used by R. L. Moore in proving Theorem 5 of his paper Concerning triods in the plane and the junction points of plane continua (loc. cit.) that no plane continuum contains an uncountable collection of mutually exclusive compact junction continua of itself.

The University of TeXas

* For the case where $M$ lies in the plane, this corollary is closely related to Theorem 2 of my paper Concerning accessibility in the plane and regular accessibility in $n$ dimensions, this Bulletin, vol. 34 (1928), pp. 504510.

† See R. L. Moore, Concerning triodic continua in the plane, to appear in Fundamenta Mathematicae. By an analog of a triod is meant a continuum which differs from a triod only in that it emanates from a continuum instead of from a point. 